\title{
THE ANOMALOUS COSMIC-RAY COMPONENT
}

\author{
A. C. Cummings and E. C. Stone \\ California Institute of Technology, Pasadena, CA 91125 USA
}

This brief report is intended to update the "anomalous component" section of the summary report of the galactic cosmic-ray working group (Mewaldt et al., 1987), which was drafted at the March 1987 Workshop on the Interplanetary Charged Particle Environment at the Jet Propulsion Laboratory. The description of the spectrum of the anomalous cosmic-ray component is contained in section 3.3 of that report. That description is based on data analyzed through day 310 of 1986 , and in it we proposed that the energy spectrum of the various species of the anomalous component could be derived by scaling from two generic spectra. Two generic spectra were required because the energy spectrum of the anomalous component changed shape near the time of the solar magnetic field reversal in 1980. These two generic spectra are shown in Figure 2 of the summary report.

We also indicated in the summary report that "it remains to be seen, however, whether the spectrum over the next few years will maintain this new shape or will return to its $1972-1977$ shape." Therefore, we have continued to monitor the energy spectrum to see whether or not the new spectral shape is truly characteristic of the new magnetic polarity epoch of the Sun. We now find evidence that it is not.

In Figure 1 we show fourteen 52-day average composite anomalous spectra from the middle of 1985 to the latest available data. The last four spectra (periods 11-14) have been added after the Workshop results were incorporated into the summary report. The dashed line is the spectrum from the last solar minimum period, normalized to the current spectrum at higher energies. It appears that the spectrum changed after period 10 . For example, from period 10 to 14 the peak energy shifts by near-

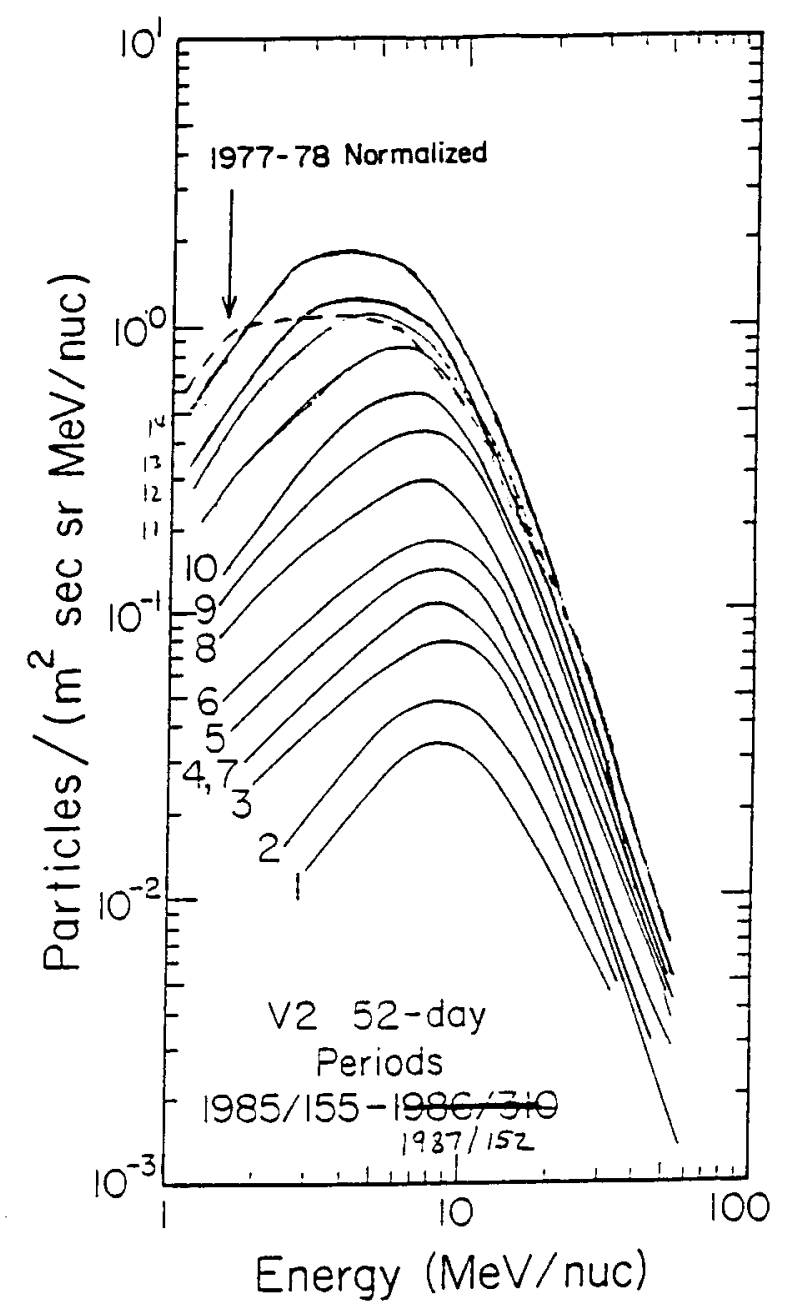

Figure 1. 
ly a factor of 2 to lower energies. This peak energy is still higher than at the last solar minimum, by $\sim 30$ to $50 \%$, and the spectrum appears to be somewhat differently shaped. However, since the recent changes have been rapid, we caution that we must still wait to see how the spectrum evolves over the next year or so before we can make definitive statements about the spectral shape of the anomalous component. Certainly, it appears incorrect to say that there are only two generic shapes for the spectrum. A more accurate description at this time would be: it appears that the spectral shape changed in $\sim 1980$, remained approximately unchanged for $\sim 6$ years, and is now undergoing a rather rapid evolution back toward the shape it had in 1977-78. For future updates, please contact the authors.

Acknowledgements. We are grateful for the contributions of R. E. Vogt in his tenure as Principal Investigator for the Cosmic Ray System (CRS) on the Voyager spacecraft. We also appreciate the contributions of the other Voyager CRS team members at Caltech and the Goddard Space Flight Center. This work was supported in part by NASA under contract NAS 7-918 and grant NGR 05-002-160.

\section{References}

Mewaldt, Cummings, Adams, Jr., Evenson, Fillius, Jokipii, McKibben, and Robinson, Toward a descriptive model of galactic cosmic rays in the heliosphere, Summary report of the galactic cosmic-ray working group of the Workshop on the Interplanetary Charged Particle Environment, JPL, March, 1987. 